\title{
Intramolecular vibrational energy redistribution from a high frequency mode in the presence of an internal rotor: Classical thick-layer diffusion and quantum localization
}

\author{
Paranjothy Manikandan and Srihari Keshavamurthy \\ Department of Chemistry, Indian Institute of Technology, Kanpur, India, 208016
}

\begin{abstract}
We study the effect of an internal rotor on the classical and quantum intramolecular vibrational energy redistribution (IVR) dynamics of a model system with three degrees of freedom. The system is based on a Hamiltonian proposed by Martens and Reinhardt (J. Chem. Phys. 93, 5621 (1990).) to study IVR in the excited electronic state of para-fluorotoluene. We explicitly construct the state space and show, confirming the mechanism proposed by Martens and Reinhardt, that an excited high frequency mode relaxes via diffusion along a thick layer of chaos created by the low frequency-rotor interactions. However, the corresponding quantum dynamics exhibits no appreciable relaxation of the high frequency mode. We attribute the quantum suppression of the classical thick-layer diffusion to the rotor selection rules and, possibly, dynamical localization effects.
\end{abstract}

Intramolecular vibrational energy redistribution (IVR) has been the focus of several theoretical and experimental studies for the past few decades $1,2,3,4,5$. An important challenge, from the viewpoint of prediction and control of reaction dynamics, is to understand the influence of specific structural features (functional groups) of a molecule on the IVR dynamics. Several studies $6,7,8,9,10,11,12,13,14,15$ suggest that the early stages of IVR can indeed be described from such a moietyspecific i.e., local viewpoint. In particular, starting with the experiments by Parmenter and coworkers $10,11,16,17$, the role of large amplitude motions involving internal ro-

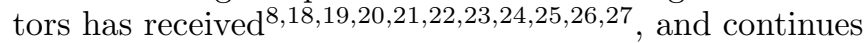
to receive $28,29,30,31,32$, considerable attention in the literature.

In this work we focus on a mechanism, suggested by Martens and Reinhardt ${ }^{26}$ and inspired by the observations of Parmenter and coworkers ${ }^{10,16}$, for the accelarated IVR rates among the ring modes in para-fluorotoluene $(\mathrm{pFT})$ in comparison to a similar molecule without the methyl rotor (para-difluorobenzene). The essential features of the proposed mechanism ${ }^{26}$, based on detailed classical dynamical studies of a model Hamiltonian for pFT incorporating the methyl rotor, five of the lowest frequency ring normal modes, and important ring-rotor couplings, can be illustrated using the 'minimal' Hamiltonian

$$
\begin{aligned}
H & =\frac{1}{2} \sum_{j=1,2}\left(P_{j}^{2}+\omega_{j}^{2} Q_{j}^{2}\right)+B P_{\phi}^{2}+\frac{V_{0}}{2}[1+\cos 6 \phi] \\
& +\sum_{j=1,2} \alpha_{j} Q_{j} \sin 3 \phi
\end{aligned}
$$

In the above, three degrees of freedom Hamiltonian, the ring normal modes 1 , and $2\left(\omega_{1}<\omega_{2}\right)$ are coupled with the hindered rotor mode but the oscillators are not directly coupled to each other. Setting $\alpha_{1}\left(\alpha_{2}\right)=0$ one obtains two degrees of freedom subsystem describing the high (low) frequency mode-rotor coupling. The subsystem dynamics can be analyzed as follows. A resonance $\omega_{j}=3 \omega_{r}$, driven by the coupling terms in Eq. (11), occurs at $P_{\phi}^{(j)} \approx \pm \omega_{j} / 6 B$ and leads to quasiperiodic exchange of energy between the oscillator and the rotor. However, if in addition the condition $P_{\phi}^{(j)} \sim \sqrt{V_{0} / B}$ is satisfied then the $\omega_{j}=3 \omega_{r}$ resonance can overlap with the hindered rotor separatrix, $E_{r}=V_{0}$, generating large scale stochasticity in the phase space leading to a rapid relaxation of the oscillator energy. Thus one anticipates that if at a given energy the low frequency-rotor subsystem $\left(\alpha_{2}=0\right.$, "bath") has a strongly chaotic phase space then an excited low frequency mode will relax rapidly. On the other hand at the same energy the high frequency-rotor subsystem $\left(\alpha_{1}=0\right)$, typically, would have a near-integrable phase space and hence an excited high frequency mode will not relax.
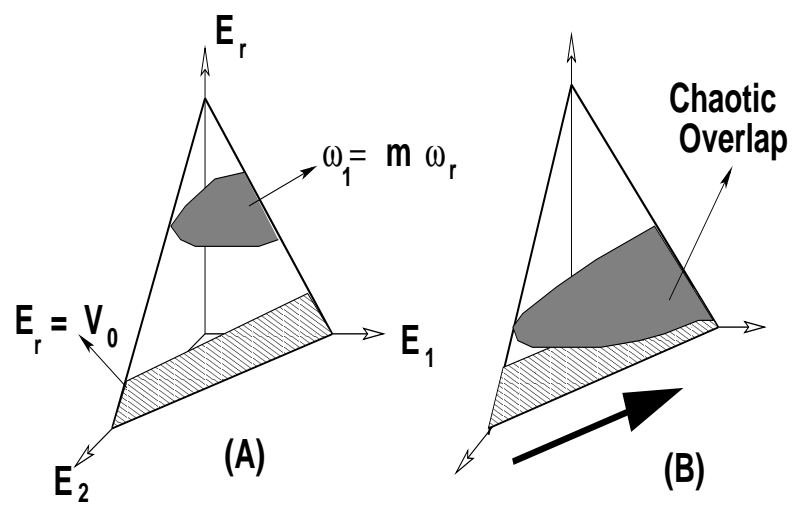

FIG. 1: Illustrating the mechanism for relaxation of a high frequency mode (2) according to Martens and Reinhardt ${ }^{26}$. In (A) the frequency $\omega_{1}$ is sufficiently high such that the $m: 1$ resonance between the bath (1) and the rotor $(r)$ does not overlap with the hindered rotor separatrix $E_{r}=V_{0}$. On the other hand, in case (B) the bath frequency is low enough for an overlap resulting in large scale stochasticity. This band of chaos now leads to the relaxation of the high frequency system over long time scales.

Interestingly, Martens and Reinhardt observed 26 that coupling the subsystems i.e., $\alpha_{1}, \alpha_{2} \neq 0$ leads to a slow relaxation of the excited high frequency mode despite the lack of resonance overlap. It was suggested ${ }^{26}$ that this could be due to a slow diffusion along the thick layer of chaos created by the bath subsystem (cf. Fig. 1B). 
Evidence for such a "stochastic pumping" mechanism ${ }^{33}$ was provided by modifying the system so that the bath dynamics is no longer strongly chaotic as sketched in Fig. 11A. Clearly, such a mechanism would lead to extensive mixing of all the ring modes and therefore support the experimental observations. However, there are crucial questions, given the debate about the precise role of the rotor ${ }^{8.11 .20}$, that have not yet been answered. Firstly, the Hamiltonian used in the original studies involved five of the ring modes and the hindered rotor. Is it possible that the 'minimal' Hamiltonian in Eq. (1) is enough to capture the main mechanisms? Secondly, can one explicitly show the existence of the thick-layer diffusion and understand the nature of this diffusion? Finally, is the mechanism valid quantum mechanically? The last issue is crucial, as was also hinted by Martens and Reinhardt, due to strict rotor selection rules $\frac{16,25}{2}$ and the possibility that quantum mechanics might localize the long time classical transport. In this work we report some of our preliminary results which provide answers to the questions posed above.

We consider the Hamiltonian in Eq. (1) with the oscillator frequencies $\omega_{1}=110 \mathrm{~cm}^{-1}, \omega_{2}=359 \mathrm{~cm}^{-1}$, rotor barrier $V_{0}=34 \mathrm{~cm}^{-1}$, and methyl rotational constant $B=4.65 \mathrm{~cm}^{-1}$. The parameters are identical to that of the earlier work ${ }^{26}$ except that only the lowest and the highest frequency modes have been retained 34 . An ensemble of classical trajectories with fixed actions $\left(J_{1}, J_{2}, P_{\phi}\right)$ and random phases of the oscillators $\left(\theta_{1}, \theta_{2}\right)$ and the rotor $(\phi)$ are generated to model initially excited nonstationary zeroth-order quantum states $\left|v_{1}, v_{2}, v_{r}\right\rangle$. In Fig. 2 we show the results for a representative case of the excited high frequency oscillator corresponding to the zeroth-order quantum state $|0,5,1\rangle$. Trajectories are propagated for $100 \mathrm{ps}$ and in Fig. 2 the ensemble averaged mode energies are shown as a function of time. It is clear that the high frequency mode relaxes over the long timescale with the energy being gained by the low frequency mode and the rotor. In fact the subsystem rotor surface of sections shown in Fig. 2 correspond to $H=E_{051}^{0} \approx 2034 \mathrm{~cm}^{-1}$ and suggest that the high frequency mode should not relax. Thus the energy flow out of mode 2 in Fig. $2 \mathrm{a}$ is a three degree of freedom effect and identical to the observations made by Martens and Reinhardt in their six degrees of freedom Hamiltonian 26 .

Is the long time energy relaxation of the mode 2 seen in Fig. 22a due to the thick-layer diffusion mechanism suggested earlier ${ }^{26}$ ? To this end we study the classical IVR dynamics by explicitly constructing the zeroth-order action space $\left(J_{1}, J_{2}, P_{\phi}\right)$ i.e., state space. A set of initial actions were chosen by fixing the total energy $H=E_{051}^{0}$ and angles $\left(\theta_{1}, \theta_{2}, \phi\right)=(0,0,0)$. These set of initial conditions are then propagated to $T=100 \mathrm{ps}$ and the diffusion constants

$$
d_{k}(T)=\frac{1}{T} \int_{0}^{T}\left|\Omega_{k}(t)-\bar{\Omega}\right| d t
$$

for $k=1,2, r$ are determined. The $\Omega_{k}$ are the local non-

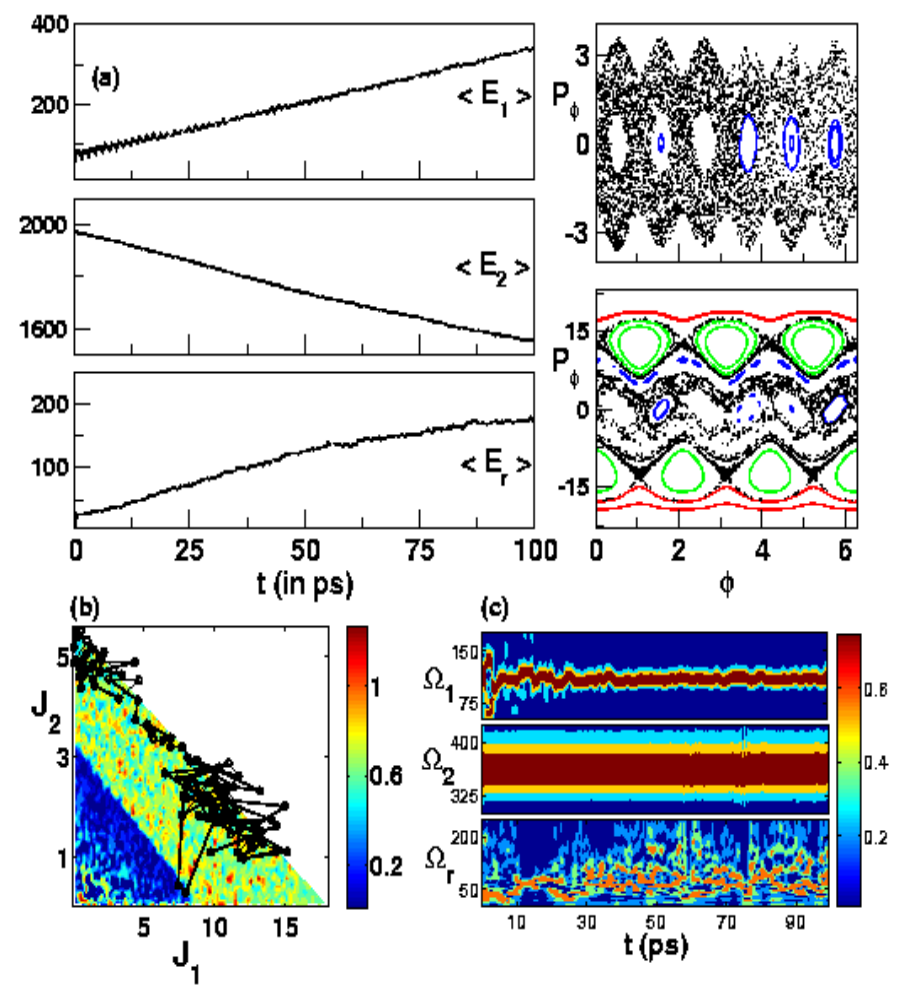

FIG. 2: (Color online) (a) Classical average mode energies (in $\mathrm{cm}^{-1}$ ) versus time for an ensemble of trajectories with initial conditions corresponding to $\left|v_{1}, v_{2}, v_{r}\right\rangle=|0,5,1\rangle$. The coupling constants ${ }^{34}$ are $\alpha_{1}=-3.14 \mathrm{~cm}^{-1} / \AA$ and $\alpha_{2}=2.3$ $\mathrm{cm}^{-1} / \AA$. The $\left(\phi, P_{\phi}\right)$ surface of sections, at the zeroth-order energy $E_{051}^{0}=2034 \mathrm{~cm}^{-1}$, for the two degrees of freedom subsystems obtained by decoupling the high and the low frequency modes respectively are also shown. The 3:1 resonance islands (green) and the rotor (blue) regions are clearly visible in the 2- $r$ subsystem. (b) The $\left(\theta_{1}, \theta_{2}, \phi\right)=(0,0,0)$ slice of the state space at $E_{051}^{0}$ is shown and exhibits regions with large and small diffusions (in $\mathrm{ps}^{-1}$ ). Note the existence of a chaotic band which should be compared to the schematic shown in Fig. 1B. A representative trajectory with large diffusion along the chaotic band is also shown in (b) with the circles representing 1 ps intervals. (c) The frequency content of the representative trajectory in (b) confirms the chaotic behaviour of the rotor-low frequency subsystem. See text for details.

linear frequencies extracted using a wavelet-based timefrequency approach ${ }^{35}$. The sum of the $d_{k}$ 's is then associated with each trajectory (point in the state space) and hence the regular (low diffusion) and chaotic (high diffusion) regions are identified. In Fig. $2 \mathrm{~b}$ the state space is shown as a projection onto the $\left(J_{1}, J_{2}\right)$ plane and the band of stochasticity, due to the mode 1 and rotor interaction as sketched in Fig. 1 B , is clearly seen. Several classical trajectories corresponding to $|0,5,1\rangle$ were propagated for $100 \mathrm{ps}$ and a variety of diffusive behaviours were seen. In Fig. 2b we show a typical high diffusion trajectory superimposed on the state space which clearly shows the diffusion along the thick layer of chaos. 
This establishes the mechanism proposed by Martens and Reinhardt for the minimal Hamiltonian in Eq. (11). Moreover, to the best of our knowledge, this is the first time that the thick-layer diffusion has been explicitly shown in a molecular system.
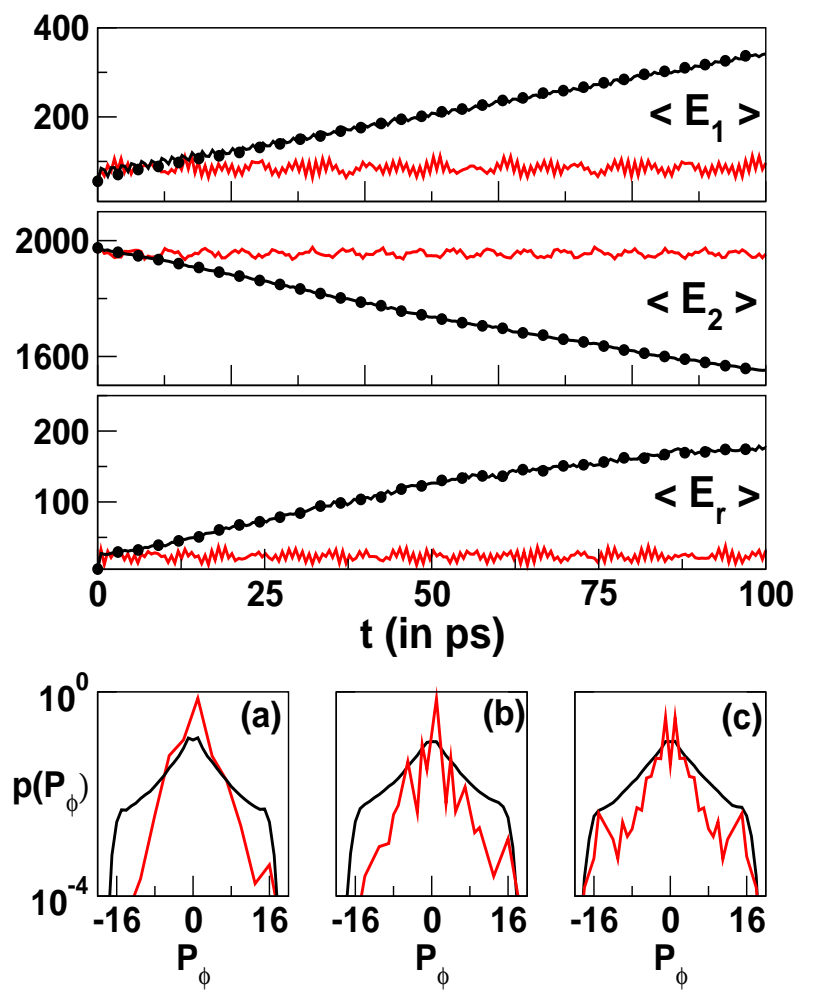

FIG. 3: (Color online) Quantum average mode energes (red) compared to the classical (black symbols) analogs shown in Fig. 2a. The bottom panels compare the classical (thick black) and quantum (red) rotor angular momentum distributions. (a) Original system (cf. Eq. 11) indicates localization of the quantum $p\left(P_{\phi}\right)$ as compared to the classical case. In (b) and (c) weak couplings between mode 1 and the rotor of the form $\beta Q_{1} \sin 2 \phi$ and $\beta Q_{1} \sin \phi$ with $\beta=-1 \mathrm{~cm}^{-1} / \AA$ respectively are added to the original system in order to break the $\Delta v_{r}= \pm 3$ selection rule in (a).

Certain features of the dynamics that are inherently due to the system having three or more degrees of freedom are worth pointing out at this stage. A careful look at the trajectory shown in Fig. $2 \mathrm{~b}$ reveals that for nearly $15 \mathrm{ps}$ there is very little energy relaxation in $E_{2}$. In our computations this feature was common to a large number of trajectories. A preliminary understanding of the $\sim 15$ ps 'incubation' time can be gained by the timefrequency analysis ${ }^{35}$ of the classical dynamics. In Fig. 2 r the results for the representative trajectory undergoing thick-layer diffusion in the state space are shown as spectrograms i.e., the distribution of the local frequency amplitudes as a function of time. One can see that during the incubation time the low frequency mode and the rotor exhibit strong coupling and chaotic dynamics while the high frequency mode is decoupled. At longer times perturbations in the local frequency associated with the mode 2 are clearly visible. The rotor phase space shown in Fig. 2f for the bath indicates a thin layer of chaos which is insuffcient to drive the energy relaxation of the high frequency mode. Consequently we interpret the incubation time as the time required for the low frequency-rotor subsystem to generate the thick layer of chaos. Incidentally, we remark here that Martens and Reinhardt arrive at nearly the same timescale for the high-frequency cutoff in the spectral density associated with the random force generated by the chaotic bath dynamics. After the incubation time the trajectory rapidly moves along the chaotic layer resulting in the energy flowing into the low frequency mode and the rotor. At longer times the trajectory once again performs diffusive motion about specific regions in the state space.

We now address the crucial issue of wether the quantum dynamics of Eq. (1) would also exhibit the thicklayer diffusion leading to the relaxation of the excited high frequency mode. Surprisingly, and as far as we can tell, there has been no attempt to confirm the mechanism proposed by Martens and Reinhardt in the quantum domain. We note, however, that Leitner and Wolynes have studied $\frac{36,37}{3}$ the effect of quantization on the weak stochastic pump model of Arnol'd diffusion. In this case, involving anharmonic oscillators, it was found that the quantization limits the extent of diffusion. Does this imply a similar localization for the quantum dynamics of our minimal Hamiltonian? To investigate this possibility we have performed detailed quantum studies of the IVR dynamics of our system. A converged basis set, using direct product of harmonic oscillator and free rotor basis functions, was employed in all the studies. In Fig. 3 we show the quantum average mode energies $\left\langle E_{k}\right\rangle$ for the zeroth-order state $|0,5,1\rangle$ and compare with the classical results of Fig. 2, It is clear from the figure that quantum mechanically the high frequency mode does not relax even on the $100 \mathrm{ps}$ timescale - infact a beating pattern is observed. It is important to mention that results of further studies (not shown here) on the quantum dynamics including the rest of the ring modes, as in the original study $y^{26}$, again indicate little to no energy flow out of the high frequency mode. Thus, it appears that the classical thick-layer diffusion is completely suppressed in the corresponding quantum system.

There can be a couple of reasons for the observed quantum behaviour. The first, as already alluded to earlier, could be due to a genuine quantum localization of the thick-layer diffusion. Consequently, relevant eigenstates of Eq. (1) were investigated and none of them displayed a strong delocalization along the chaotic layer. Nevertheless, the fact that the high frequency mode does not relax even upon the inclusion of additional ring modes points to an insufficient density of states in the chaotic layer. One other possibility has to do with the fact that quantum mechanically there is a strict rotor selection rule $\Delta v_{r}= \pm 3$ due to the $Q \sin 3 \phi$ coupling in Eq. (1). Thus the low frequency-rotor coupling cannot lead to the pop- 
ulation of specific rotor states as opposed to the situation in the classical dynamics. The consequences can be clearly seen in Fig. 3 a where the quantum rotor momentum distribution 38

$$
p\left(v_{r}=\hbar P_{\phi}\right)=\frac{1}{T} \int_{0}^{T} d t \sum_{v_{1} v_{2}}\left|\left\langle v_{1} v_{2} v_{r}\left|e^{-i H t / \hbar}\right| 0,5,1\right\rangle\right|^{2}
$$

is compared with the corresponding classical distribution $^{26} p\left(P_{\phi}\right)$ at $T=100$ ps. The quantum distribution is localized and the asymmetric form of the envelope is due to the selection rule. In order to assess the importance of the rotor selection rule to the observed quantum localization weak coupling terms between the low frequency mode and the rotor of the form $\beta Q_{1} \sin 2 \phi$ and $\beta Q_{1} \sin \phi$ were introduced in Eq. (1) with $\beta=-1 \mathrm{~cm}^{-1} / \AA$. The resulting quantum and classical $p\left(P_{\phi}\right)$ are compared in Fig. 3b and Fig. 3. respectively. Although one observes population of higher momentum states, the quantum distribution is still narrower than the classical distribution. At the same time even for the modified cases no energy relaxation of the high frequency oscillator is observed. Therefore, given the lack of a sufficient density of states in the chaotic layer, rotor selection rule might not be the sole factor responsible for the quantum localization. The classical-quantum correspondence of $p\left(P_{\phi}\right)$ have been studied earlier ${ }^{38}$ in the context of periodically kicked one degree of freedom systems. In our case the system has three degrees of freedom and the nature of partial barriers is not yet clear. Further work, in addition to a careful study of the dependence of energy diffusion and quantum eigenstates on $\hbar$, is required to provide a firm answer and such studies are currently underway.

The authors acknowledge Profs. Gerrit Groenenboom, David Leitner, and Brooks Pate for useful discussions and suggestions.
${ }^{1}$ K. K. Lehmann, G. Scoles, and B. H. Pate, Annu. Rev. Phys. Chem. 45, 241 (1994).

2 D. J. Nesbitt and R. W. Field, J. Phys. Chem. 100, 12735 (1996).

3 J. Keske, D. A. McWhorter, and B. H. Pate, Int. Rev. Phys. Chem. 19, 363 (2000).

4 M. Gruebele and P. G. Wolynes, Acc. Chem. Res. 37, 261 (2004).

5 T. Uzer, Phys. Rep. 199, 73 (1991).

${ }^{6}$ E. L. Sibert, W. P. Reinhardt, and J. T. Hynes, J. Chem. Phys. 81, 1115 (1984).

7 A. McIlroy and D. J. Nesbitt, J. Chem. Phys. 92, 2229 (1990); 101, 3421 (1994).

8 J. E. Gambogi, L'Esperance, K. K. Lehmann, B. H. Pate, and G. Scoles, J. Chem. Phys. 98, 1112 (1993).

9 J. B. Hopkins, D. E. Powers, and R. E. Smalley, J. Chem. Phys. 71, 3886 (1979); D. E. Powers, J. B. Hopkins, and R. E. Smalley, ibid. 72, 5721 (1980); 74, 5971 (1981).

10 C. S. Parmenter and B. M. Stone, J. Chem. Phys. 84, 4710 (1986).

11 D. B. Moss and C. S. Parmenter, J. Chem. Phys. 98, 6897 (1993).

12 J. S. Hutchinson, W. P. Reinhardt, and J. T. Hynes, J. Chem. Phys. 79, 4247 (1983).

13 E. R. Th. Kerstel, K. K. Lehmann, T. F. Mentel, B. H. Pate, and G. Scoles, J. Phys. Chem. 95, 8282 (1991).

14 G. A. Bethardy, X. Wang, and D. S. Perry, Can. J. Chem. 72, 652 (1994).

15 G. T. Fraser, B. H. Pate, G. A. Bethardy, and D. S. Perry, Chem. Phys. 175, 223 (1993).

16 D. B. Moss, C. S. Parmenter, and G. E. Ewing, J. Chem. Phys. 86, 51 (1986).

17 P. J. Timbers, C. S. Parmenter, and D. B. Moss, J. Chem. Phys. 100, 1028 (1994).

18 V. A. Walters, S. D. Colson, D. L. Snavely, K. B. Wiberg, and B. M. Jamison, J. Phys. Chem. 89, 3857 (1985).

19 P. Hoffman, R. B. Gerber, M. A. Ratner, L. C. Baylor, and E. Weitz, J. Chem. Phys. 88, 7434 (1988).
20 J. S. Baskin, T. S. Rose, and A. H. Zewail, J. Chem. Phys. 88, 1458 (1988).

21 J. H. Frederick and G. M. McClelland, J. Chem. Phys. 84, 4347 (1986).

22 L. G. Spears and J. S. Hutchinson, J. Chem. Phys. 88, 240 (1988); 88, 250 (1988).

23 B. G. Sumpter, C. C. Martens, and G. S. Ezra, J. Phys. Chem. 92, 7193 (1988) and references therein.

24 H. W. Schranz and M. A. Collins, J. Chem. Phys. 98, 1132 (1992); 100, 2089 (1993); 101, 307 (1994).

25 J. T. Vivian, S. A. Lehn, and J. H. Frederick, J. Chem. Phys. 107, 6646 (1997).

26 C. C. Martens and W. P. Reinhardt, J. Chem. Phys. 93, 5621 (1990).

27 Z. Q. Zhao, C. S. Parmenter, D. B. Moss, A. J. Bradley, A. E. W. Knight, and K. G. Owens, J. Chem. Phys. 96, 6362 (1992).

28 J. A. Davies, K. L. Reid, M. Towrie, and P. Matousek, J. Chem. Phys. 117, 9099 (2002).

29 D. R. Borst and D. W. Pratt, J. Chem. Phys. 113, 3658 (2000).

30 C. J. Hammond, V. L. Ayles, D. E. Bergeron, K. L. Reid, and T. G. Wright, J. Chem. Phys. 125, 124308 (2006).

31 R. Pearman and M. Gruebele, Z. Phys. Chemie 214, 1439 (2000).

32 C. K. Nandi, M. K. Hazra, and T. Chakraborty, J. Chem. Phys. 121, 7562 (2004).

33 A. J. Lichtenberg and M. A. Lieberman, Regular and Stochastic Motion (Springer, New York, 1983).

34 The coupling constants $\alpha_{j}$ reported in ref. 26 appear to be too large by a factor of 100 . This has been confirmed by reproducing the results in ref. 26 and in this work the correct coupling values are used.

35 C. Chandre, S. Wiggins, and T. Uzer, Physica D 181, 171 (2003).

36 D. M. Leitner and P. G. Wolynes, Phys. Rev. Lett. 79, 55 (1997).

37 D. M. Leitner and P. G. Wolynes, Chem. Phys. Lett. 276, 
289 (1997).

38 G. Radons, T. Geisel, and J. Rubner, Adv. Chem. Phys.

LXXIII, 891 (1989). 